\title{
Detección óptica en 3D de un objeto flotante en una superficie marina agitada
}

\section{D optical detection of the floating object on agitated sea surface}

\author{
PAREDES-PEREZ, Ana Karen†*, GOLIKOV, Víctor y ALAZKI, Hussain \\ Universidad Autónoma del Carmen, Facultad de Ingeniería, México.
}

ID $1^{\text {er }}$ Autor: Ana Karen, Paredes-Perez / ORC ID: 0000-0003-1064-673X, Researcher ID Thomson: AAP-2216-2020, CVU CONACYT ID: 919218

ID $1^{\text {er }}$ Coautor: Victor, Golikov / ORC ID: 0000-0001-7241-2168, Researcher ID Thomson: AAA-8337-2019

ID $2^{\text {do }}$ Coautor: Hussain, Alazki /ORC ID: 0000-0002-1960-3624, Researcher ID Thomson: M-1706-2017

DOI: $10.35429 /$ JOTI.2020.14.4.7.16

Recibido 15 de Julio, 2020; Aceptado 30 de Diciembre, 2020

\section{Resumen}

En este artículo se realiza una comparativa entre dos detectores: el Detector Subespacial Acoplado MSD (Matched Subspace Detector) y el Detector Subespacial Acoplado Modificado MMSD (Modify Matched Subspace Detector) cuando hay una secuencia de imágenes (detección en 3D), en donde los parámetros de la superficie marina y el objeto flotado son desconocidos a priori, mediante simulación por computadora con ayuda del software computacional MATLAB. Ambos detectores están basados en la Prueba de Razón de Verosimilitud Generalizada GLRT (General Likelihood Ratio Test); este método ayuda a solucionar los problemas de detección cuando los parámetros de la superficie y el objeto son desconocidos. La superficie del mar es simulada como un proceso aleatorio Gaussiano, y el objeto flotado como un proceso determinístico desconocido a priori. Este artículo considera la dependencia de la probabilidad de detección con una probabilidad fija de falsa alarma en la diferencia entre los valores promedio de los reflejos de la superficie del mar y de un objeto flotante con diferente valor de potencia de las fluctuaciones de los reflejos del objeto y de la superficie del mar.

Detección de objeto, Superficie del mar, Procesamiento multipixel

\begin{abstract}
In this paper we realize a comparison between two detectors: Matched Subspace Detector (MSD) and Modify Matched Subspace Detector (MMSD) when there is a images secuence (3D detection), where the parameters of sea surface and the parameters of floating object are priori unknown in computer simulation, with help of computer software MATLAB. Both detectors (MSD and MMSD) are based in the General Likelihood Ratio Test (GLRT); this method helps solve detection problems when the sea surface and floating object parameters are unknown. The sea surface is simulated as a Gaussian random process, and the floating object is simulated as a priori unknown deterministic process. The paper considers the dependence of the probability of detection with a fixed probability of false alarm on the difference between the average values of reflections from the sea surface and from a floating object with different ratios of the power of fluctuations of reflections from the object and from the sea Surface.
\end{abstract}

3D detection target, sea surface, Multipixel process

Citación: PAREDES-PEREZ, Ana Karen, GOLIKOV, Víctor y ALAZKI, Hussain. Detección óptica en 3D de un objeto flotante en una superficie marina agitada. Revista de Invención Técnica. 2020. 4-14:7-16.

\footnotetext{
*Correspondencia al Autor (Correo Electrónico: 100637@mail.unacar.mx)

$\dagger$ Investigador contribuyendo como primer autor.
} 


\section{Introducción}

La detección de objetos utilizando una cámara de video ha sido de gran valor para distintas aplicaciones, como lo son los sistemas de vigilancia, navegación autónoma, monitoreo ambiental entre otros como en (Kimura, y otros, 2012) (Osorio, y otros, 2015) (Siqueira \& Machado, 2016) y (Chacon Murguia, Sandoval Rodriguez, \& Arias Enriquez, 2012).

Sin embargo, uno de los retos más grandes a los que se ha enfrentado la detección de objetos con video cámara es cuando el objeto se encuentra en una superficie fluctuante, y sus parámetros son desconocidos a priori. Existen muchas investigaciones sobre la detección de objetos en una superficie fluctuante; algunos se basan en la eliminación del fondo fluctuante (Blostein \& Huang, 1991), procesamiento multi-espectral (Gnanadesikan, 2011) e híperespectral (Healey \& Slater, 1999) y el seguimiento de multitrama (Wang, Liao, Xiong, \& Zhang, 2014).

Sin embargo, todas estas tienen en común que asumen que la potencia de fondo bajo hipótesis estadística nula $H_{0}$ es igual bajo la hipótesis estadística alternativa $H_{1}$.En los sistemas ópticos, es común que la covarianza bajo ambas hipótesis sea la misma, pero la varianza es distinta. La solución a este problema es propuesta es (Golikov, Lebedeva, Castillejos Moreno, \& Ponomaryov, 2011).

El detector propuesto MMSD contiene un término adaptativo correctivo en el umbral; este término, disminuye el valor del umbral estimado automáticamente, y por lo tanto, aumenta la probabilidad de detección. Sin embargo, el detector MMSD, aún no ha sido investigado completamente.

En la práctica, el posicionamiento o localización del objeto flotado es un valor desconocido a priori, además, las reflexiones del mar pueden ser diferentes, por lo que hay que investigar la eficiencia de los detectores MSD y MMSD para realizar la detección con errores mínimos posibles.
En este artículo abordamos el problema de detección 3D de un objeto flotado en una superficie fluctuante es una secuencia de imágenes, utilizando datos de simulación con ayuda del software MATLAB, comparando el ya conocido detector MSD y el recientemente propuesto MMSD (Golikov \& Lebedeva, 2013), e investigamos la calidad de detección en el caso de variaciones en la potencia del fondo y el objeto flotado, cambios en la correlación de la superficie y ruido del canal. El principal interés es mejorar la calidad de detección en el caso de objetos flotados en una superficie fluctuante, en este caso, la superficie marina.

\section{Desarrollo del modelo del mar como un proceso aleatorio Gaussiano correlacionado}

Existen diferentes tipos de modelos para representar el comportamiento de las señales de la luz que se reflejan en la superficie del mar o superficie del objeto flotado, el más popular para representar este tipo de reflexiones se basa en un proceso aleatorio Gaussiano en tres dimensiones. Consideramos una señal en la salida de la cámara de video compuesta por $K$ cuadros sucesivos. La forma en la que el detector se desempeña es recibiendo una matriz de tres dimensiones, de tamaño $L \times N \times K$ donde $L$ es el número de filas, $N$ el número de columnas, y $K$ el número de cuadros de la matriz. Así mismo, se puede interpretar $L$ como primera dimensión, $N$ como segunda dimensión y $K$ como tercera dimensión.

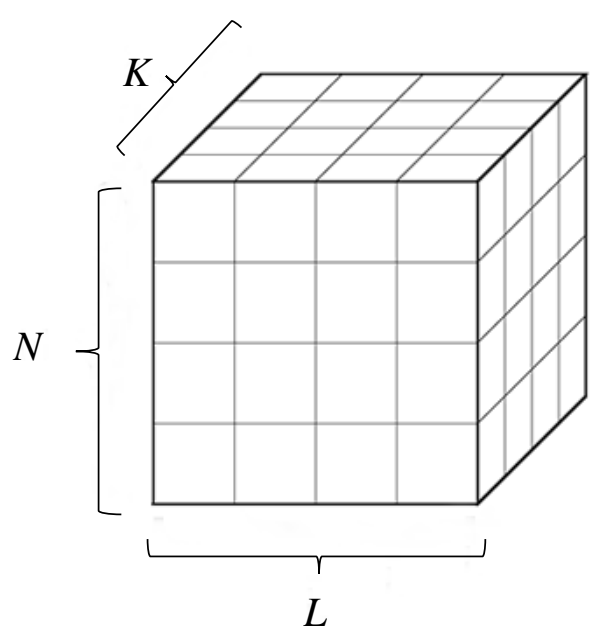

Figura 1 Ejemplo de matriz de tres dimensiones de $L$ filas, $N$ columnas y $K$ cuadros

Fuente: Edición del autor 
Las distribuciones espectrales de las señales de luz reflejadas desde la superficie del mar dependen de la velocidad del viento, normalmente tienen más componentes espectrales que las del objeto flotado. Para crear este tipo de reflexiones como un proceso aleatorio Gaussiano usamos un paquete de MATLAB y su comando randn que puede crear un proceso aleatorio con densidad de probabilidad Gaussiana en tres dimensiones. Para la representación de una secuencia de imágenes usando MATLAB se utiliza un tipo de dato llamado uint8, donde todos los valores posibles de los pixeles en las imágenes pueden tomar valores enteros entre 0 y 255 , donde 0 es completamente negro, y 255 es completamente blanco. Este tipo de dato ayudará a representar algunos parámetros del proceso aleatorio Gaussiano como la media $\mu$, la varianza $\sigma^{2}$, y el factor de correlación corr.

Para crear un proceso correlacionado, usamos la matriz de correlación exponencial

$r_{j_{1}, j_{2}}=e^{\left(-a\left|j_{1}-j_{2}\right|\right)}$

Donde $j_{1}$ son los renglones, $j_{2}$ las columnas de una matriz de covarianza, y $a$ tiene relación con el factor de correlación corr dado por la ecuación (2)

$a=1-\operatorname{corr}$

El comando levinson de MATLAB usa la ecuación (1) con el factor de correlación preestablecido (normalmente entre 0.95 y 0.8 ) para generar coeficientes de $g$ y $a$ de dos polinomios (numerador $\mathrm{y}$ denominador). El comando filter crea un proceso Gaussiano correlacionado adecuado a los coeficientes $g$ y $a$. La fuente del proceso Gaussiano es el comando randn con media cero, varianza uno, y valores decorrelacionados. El comando filter puede transferir el proceso decorrelacionado randn a un proceso también Gaussiano con media cero, y varianza que depende de $g$ y $a$, con función de correlación según la fórmula (1) en una dimensión establecida (primera o segunda dimensión). Para obtener una imagen correlacionada simultáneamente en dos dimensiones aplicamos el comando permute para girar la imagen $90^{\circ}$ grados y con esta imagen aplicar de nuevo el comando filter.
Agregamos este proceso a un ciclo for para crear la secuencia de imágenes. Todas las imágenes son matrices de correlacionadas entre sí. Las mediciones de reflexiones usando una videocámara contienen errores adicionales, como fluctuaciones de propagación de luz, además de ruido de cuantización dentro de la videocámara. La suma de estos dos errores, es conocida como el ruido del canal. Las mediciones desde la superficie del mar siempre contienen un ruido del canal, y en este caso se estará manejando con la variable $w$ para cambiar la varianza del ruido del canal. El modelo del ruido del canal es un proceso aleatorio Gaussiano de correlacionado, con media cero, y varianza que depende de la situación del medio ambiente y el tipo de cámara. En la figura 2 se presenta un ejemplo del modelo de la superficie del mar generado con MATLAB.

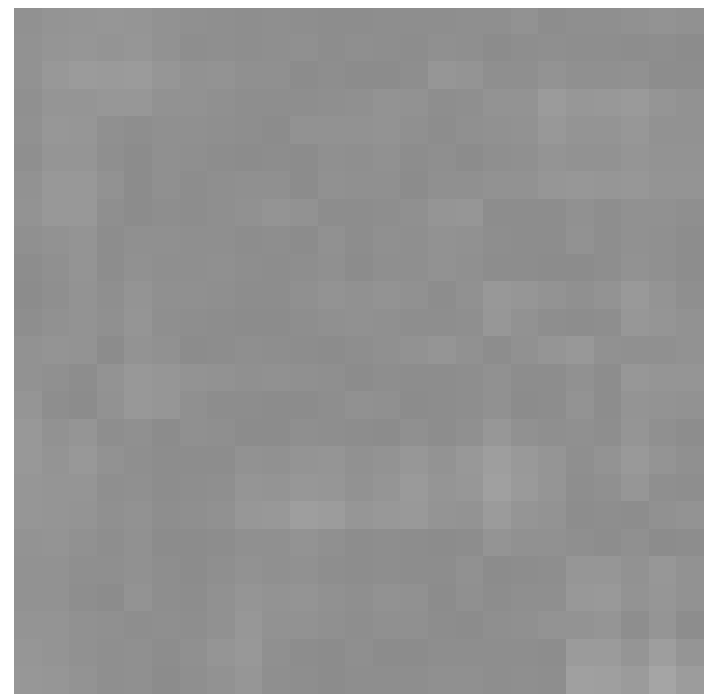

Figura 2 Modelo del mar correlacionado en primera y segunda dimensión tamaño $25 \times 25 . \mu=140, \sigma^{2}=30$, corr $=0.9, w=0.01$

Fuente: Edición del autor, Figura extraída de MATLAB

\section{Desarrollo del modelo del objeto flotado como un proceso determinístico desconocido a priori}

Consideramos el caso en tres dimensiones cuando el objeto flotado está dentro de un área de varios píxeles. Normalmente el objeto flotado se mueve de acuerdo con el tamaño de las olas marinas, de su propia forma, tamaño, peso, etc. Los parámetros de reflexión del objeto flotado dependen de sus oscilaciones y contienen un pequeño número de componentes espectrales, por lo tanto, el modelo del objeto flotado debe tener valores diferentes en cada imagen y a priori desconocidos. 
La mayoría de los modelos de objetos flotados se basan en un modelo lineal dado por la siguiente ecuación:

$\boldsymbol{s}=\boldsymbol{H \theta}$

Donde $\boldsymbol{s}$ es un vector de la señal del objeto flotado, $\boldsymbol{H}$ es la matriz que contiene en sus columnas los armónicos de la transformada discreta de Fourier, y $\boldsymbol{\theta}$ es un vector-columna que presenta amplitudes de las armónicas de la transformada discreta de Fourier. La señal $\boldsymbol{s}$ es una suma de componentes espectrales de los armónicos $\boldsymbol{H}$ con amplitudes $\boldsymbol{\theta}$ a priori desconocidas. La matriz $\boldsymbol{H}$ está definida de acuerdo con la siguiente ecuación:

$\boldsymbol{H}=\left[\begin{array}{cccc}1 & 1 & \cdots & 1 \\ z_{0} & z_{1} & \cdots & z_{n} \\ \vdots & \vdots & \cdots & \vdots \\ z_{0}^{N-1} & z_{1}^{N-1} & \cdots & z_{n}^{N-1}\end{array}\right]$

Donde $z_{n}^{i}=\exp \left(\frac{j 2 \pi i n}{N}\right) ;$ el subíndice $n$ indica el número de columna de la matriz $\boldsymbol{H}$ y el número de armónico $n=1,2, \ldots, p$. La variable $i$ indica el número de fila de la matriz $\boldsymbol{H}$ y el valor recibido en tiempo discreto $i=$ $0,1,2, \ldots, N-1$. $\quad N$ es el número de valores en cada columna y también el número de valores recibidos por el detector, $j=\sqrt{-1}$. En este artículo asumimos que el valor de $p$ siempre será menor que $N$, ya que el objeto flotado es un objeto sólido, por lo tanto, sus fluctuaciones no contienen frecuencias altas, en cambio, la superficie marina se trata de líquido, por lo tanto, su frecuencia de luz reflejada puede ser lo suficientemente alta.

La ecuación (3) también puede ser representada de la siguiente manera:

$\boldsymbol{s}=\sum_{n=1}^{p} \boldsymbol{\theta}_{n} \boldsymbol{h}_{n}$

Donde $p$ indica el número máximo de la frecuencia (máximos armónicos) del modelo del blanco, $\boldsymbol{\theta}_{n}$ es el coeficiente de amplitud de cada armónico de Fourier, y $\boldsymbol{h}_{n}$ son las columnas que hacen la matriz $\boldsymbol{H}$ del modelo lineal $\boldsymbol{H}=$ $\left\{\boldsymbol{h}_{1}, \boldsymbol{h}_{2}, \cdots, \boldsymbol{h}_{n}\right\}$.
Fue desarrollado en MATLAB un programa adecuado a las ecuaciones (3), (4) y (5). El resultado de este programa fue una secuencia de cinco imágenes consecutivas de tamaño $10 \times 10$, como se muestra en la figura 3

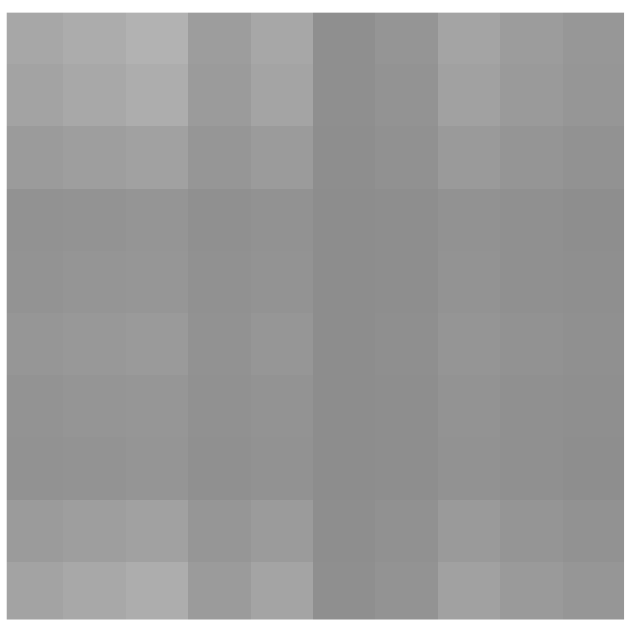

Figura 3 Modelo del objeto flotado de tamaño $10 \times 10$. $\mu_{s}=140, \sigma_{s}^{2}=70$

Fuente: Edición del autor. Figura extraída de MATLAB

El modelo del objeto está diseñado como un proceso determinístico, pero para cada muestra de $\boldsymbol{\theta}$ es diferente. El modelo del objeto flotado es insertado en el modelo de la superficie del mar, como se muestra en la figura 4.

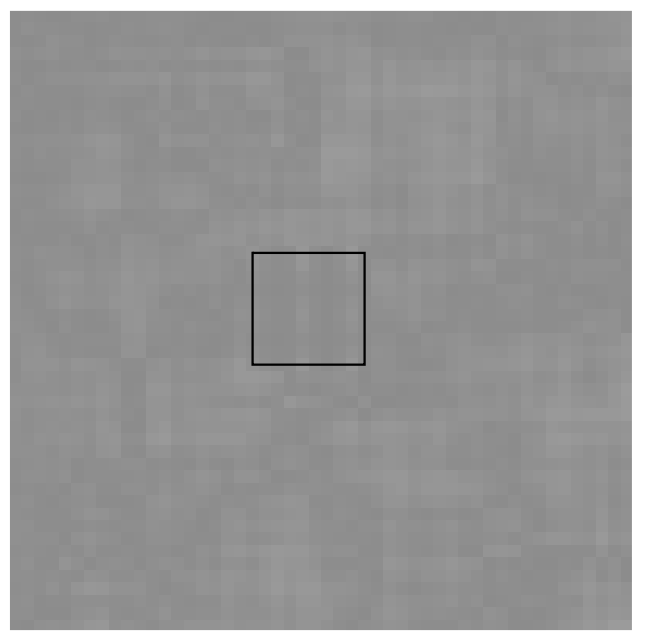

Figura 4 Modelo del objeto flotado de tamaño $10 \times 10$, dentro del modelo de la superficie del mar de tamaño $50 \times 50$. Modelo del mar: $\mu=130, \sigma^{2}=10$, $\operatorname{corr}=$ 0.9. Modelo del objeto flotado: $\mu_{s}=130, \sigma_{s}^{2}=10$

Fuente: Edición del autor. Figura extraída de MATLAB

Para diferenciar el modelo de la superficie del mar del modelo del objeto flotado fue colocado un recuadro negro que rodea el modelo del objeto. Cabe señalar que para procesos determinísticos no se puede calcular la función de densidad de probabilidad, pero la media y la varianza sí pueden ser calculadas. 


\section{Planteamiento del problema y el proceso de detección}

El problema abordado aquí es la detección en tres dimensiones de un objeto multipixel en presencia de fondo en movimiento con ruido del canal. Debemos decidir entre la hipótesis nula $H_{0}$ (fondo+ruido del canal) y la hipótesis alternativa $H_{1}$ (fondo+objeto+ruido del canal):

$H_{0}: y=\boldsymbol{n}, H_{1}: y=\mu \boldsymbol{H} \boldsymbol{\theta}+\boldsymbol{n}$

Cuando la matriz de covarianza del fondo $\boldsymbol{R}$ y el parámetro ${\sigma_{0}}^{2}$ son conocidos, pero el parámetro $\boldsymbol{\theta}$ es desconocido, la detección estadística apropiada es presentada en la forma de MSD (Manolakis \& Shaw, 2002) (Scharf, 1991):

$T_{M S D}=\left(\frac{1}{N \sigma_{0}^{2}}\right) \boldsymbol{y}^{H} \boldsymbol{R}^{-1} \boldsymbol{H}\left(\boldsymbol{H}^{H} \boldsymbol{R}^{-1} \boldsymbol{H}\right)^{-1} \boldsymbol{H}^{H} \boldsymbol{Y} \boldsymbol{R}^{-1}=\left(\frac{1}{N \sigma_{0}^{2}}\right) \boldsymbol{z}^{H} \boldsymbol{P} \boldsymbol{z} \frac{{ }^{H_{1}}}{\digamma_{H_{0}}} \eta$,

Donde $\boldsymbol{z}=\boldsymbol{y} \boldsymbol{R}^{-1 / 2}, \boldsymbol{P}=\boldsymbol{H}\left(\boldsymbol{H}^{H} \boldsymbol{H}\right)^{-1} \boldsymbol{H}^{H}$ es la matriz proyector $\boldsymbol{P}$ de tamaño $N \times N$. $\boldsymbol{H}$ tiene un tamaño de $N \times p$, y sería la matriz que genera el subespacio.

Recientemente el detector ha derivado en el caso de los parámetros desconocidos $\sigma_{1}^{2}$ y $\boldsymbol{\theta}$ usando la razón de verosimilitud generalizada empleando la función de densidad de probabilidad condicional $(p d f)$ :

$T_{M M S D}(w)=\frac{\mathbf{z}^{H} \mathbf{z}}{N \sigma_{0}^{2}}-\ln \frac{\mathbf{z}^{H} \mathbf{P}^{\perp}}{(N-p) \sigma_{0}^{2}}-1$

Donde $\boldsymbol{P}^{\perp}=\boldsymbol{I}-\boldsymbol{P}, \quad \boldsymbol{I}$ es la matriz identidad y $\boldsymbol{P}$ la matriz proyector a subespacio de la señal $s$.

El proceso de detección se divide en dos fases: primero se calculan los umbrales que separan dos zonas: una donde se recibe la decisión de que $H_{0}$ sea cierta, cuando la salida del detector sea menor que el umbral establecido, y la otra zona a la derecha del umbral, donde $H_{1}$ es cierta cuando la salida del detector sea mayor que el umbral establecido.

Para calcular los umbrales para cada detector, hay que elegir un espacio del modelo de la superficie marina donde no aparecerá el blanco de interés (en la parte superior izquierda de la figura 5), la cual será nuestra ventana del umbral.
El tamaño de esta ventana puede ser, por ejemplo, de $10 \times 10$. Después elegimos la ventana de análisis del mismo tamaño que puede coincidir o no con la posición del modelo del objeto. En todos los casos el modelo del objeto de tamaño $10 \times 10$ está en el centro de cada imagen. Se realiza el procedimiento de los detectores MSD y MMSD, con los valores de la superficie del mar dentro de la ventana del umbral.

Después de realizar el procedimiento, en la salida de cada detector aparecerá un valor que será guardado dentro de un acumulador. Este proceso se repetirá mil veces, por lo que el acumulador tendrá mil valores para ambos detectores cada uno. Los datos obtenidos serán ordenados en un vector-columna, de mayor a menor (de arriba hacia abajo) con el comando sort de MATLAB. Se establece la probabilidad de falsa alarma, utilizando técnicas de conteo estándar de Monte Carlo basadas en $100 / P_{f a}$, donde $P_{f a}$ es la probabilidad de falsa alarma preestablecida. En todas las simulaciones realizadas en este artículo $P_{f a}=10^{-2}$.

La segunda fase del proceso de detección se basa en un monitoreo de la secuencia de imágenes del modelo de la superficie del mar, usando una ventana de análisis de tres dimensiones $10 \times 10 \times 5$. Para tomar la decisión de si hay o no dentro de la ventana de análisis el modelo del objeto flotado cada detector realiza su procesamiento con $10 \times 10 \times$ 5 valores recibidos, y en sus salidas se forma un valor.

La ventana de análisis $10 \times 10 \times 5$ coincida con el tamaño del modelo del objeto flotado, y realizamos el proceso de detección. La cifra arrojada por el detector se compara con el umbral para tomar la decisión. Cuando esta cifra es mayor que el umbral el detector toma decisión que dentro de la ventana de análisis hay el objeto de interés. Cuando esta cifra es menor que el umbral, el detector toma la decisión que dentro de la ventana de análisis no hay el objeto de interés. 


\section{Resultados de detección de dos detectores elegidos a través de los modelos}

En esta sección observaremos el resultado de la estimación de la calidad de detección para dos detectores al cambiar el factor de correlación del fondo $\operatorname{corr}$, la intensidd del ruido del canal $w$, el coeficiente de sensitividad del segundo término del algoritmo MMSD, la posición de la ventana de análisis (coincide o no completamente con el objeto) y la relación señal-a-fondo SBR. El sistema de simulación de los detectores MSD y MMSD mantiene la probabilidad de falsa alarma igual a 0.01. Presentamos el caso cuando el coeficiente de variación del modelo de la superficie del mar y el modelo del objeto flotado es diferente.

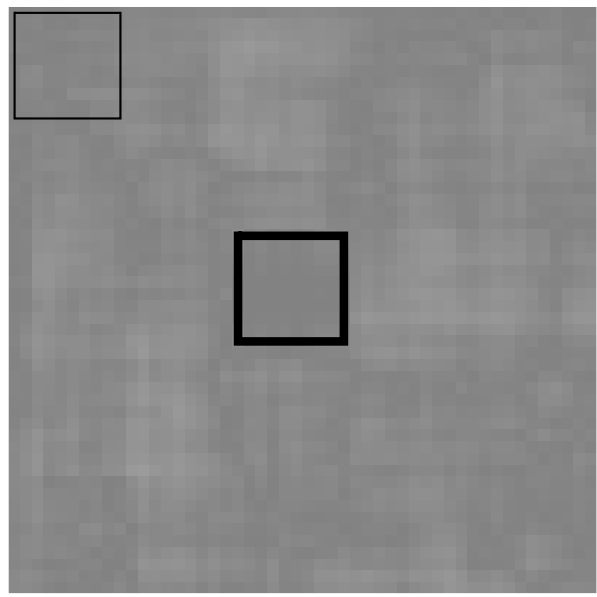

Figura 5 Modelo del objeto flotado (la primera imagen) de tamaño $10 \times 10$, dentro del modelo de la superficie del mar de tamaño $50 \times 50$, con ventana de análisis. Modelo del mar: $\mu=140, \sigma^{2}=16$, corr $=0.9$. Modelo del objeto flotado: $\mu_{s}=140, \sigma_{s}^{2}=3$

Fuente: Edición del autor. Figura extraída de MATLAB

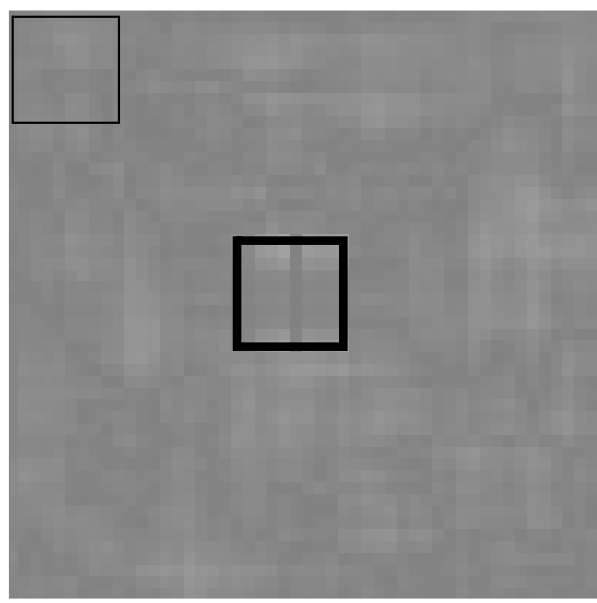

Figura 6 Modelo del objeto flotado (primera imagen) de tamaño $10 \times 10$, dentro del modelo de la superficie del mar de tamaño $50 \times 50$, con ventana de análisis. Modelo del mar: $\mu=140, \sigma^{2}=16$, corr $=0.9$. Modelo del objeto flotado: $\mu_{s}=140, \sigma_{s}{ }^{2}=18$

Fuente: Edición del autor. Figura extraída de MATLAB
En la figura 5 y 6 , la varianza del blanco es de 18 y 75 respectivamente. La media establecida del mar y del blanco en ambos casos es de 140. La varianza del mar en las dos figuras anteriores es 16. Su factor de correlación es ambas imágenes es corr $=0.9$. En el centro de las figuras 5 y 6 fue colocada la ventana de análisis de igual tamaño al blanco de interés. A continuación, mostraremos cómo afecta la calidad de detección en el caso de los modelos presentados en las figuras 5 y 6 , cambiando el factor de sensitividad del algoritmo MMSD, con ruido del canal $w=0.1$

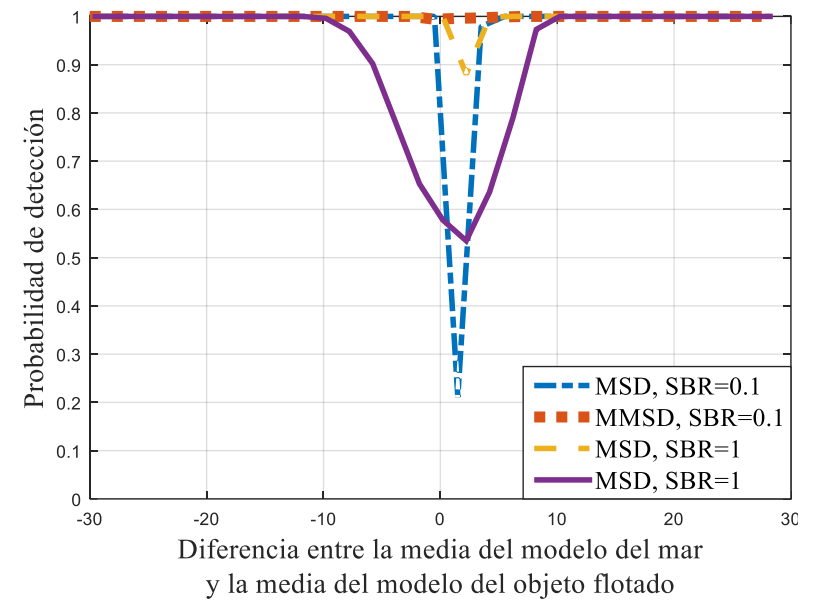

Gráfico 1 Probabilidad de detección vs diferencia entre la media del modelo del mar y la media del objeto flotado corr $=0.9, w=0.1, b=5$

Fuente: Edición del autor. Gráfico extraído de MATLAB.

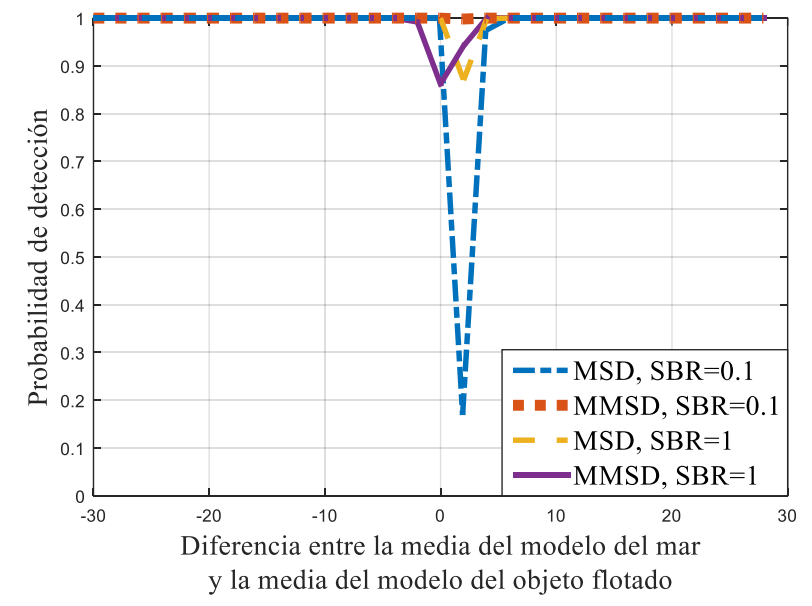

Gráfico 2 Probabilidad de detección vs diferencia entre la media del modelo del mar y la media del objeto flotado. corr $=0.9, w=0.1, b=0.5$

Fuente: Edición del autor. Gráfico extraído de MATLAB 


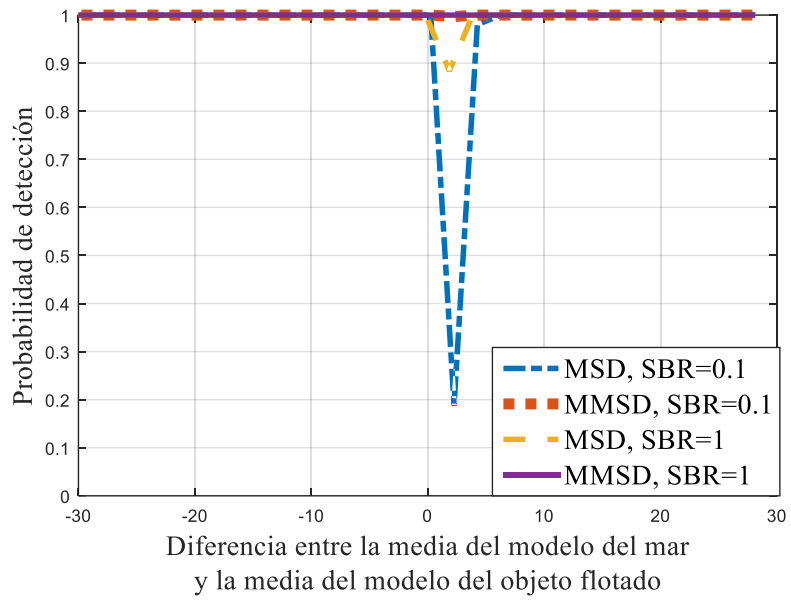

Gráfico 3 Probabilidad de detección vs diferencia entre la media del modelo del mar y la media del objeto flotado. corr $=0.9, w=0.1, b=0.05$

Fuente: Edición del autor. Gráfico extraído de MATLAB

En los gráficos 1,2 y 3 se muestra la probabilidad de detección contra la diferencia entre medias del mar y del objeto flotado; el primer par indican cómo afecta la calidad de detección de los detectores MSD y MMSD cuando la relación señal-a-fondo SBR (Signalto-background ratio) es muy pequeña, y el segundo par de curvas cuando el valor de SBR crece. También vemos cómo afecta la calidad de detección el cambio en el coeficiente de sensibilidad del MMSD. Los tres gráficos corresponden al caso de los modelos en las figuras 5 (para el primer par de curvas, $\mathrm{SBR}=0.1$ ) y 6 (para el segundo par de curvas, $\mathrm{SBR}=1)$.

Podemos observar que el algoritmo MSD es insensible a los cambios del coeficiente de sensitividad $b$, ya que este solo se encuentra en el algoritmo MMSD. Sin embargo, analizando los gráficos 1,2 y 3 , observamos que a mayor aumento del SBR, el algoritmo MSD tendrá una mejor calidad de detección cuando las medias del mar y el del objeto flotado sean aproximadamente iguales.

En el caso del algoritmo MMSD se observa que es indiferente a los cambios de $b$ siempre y cuando la relación SBR tenga valores muy pequeños, como 0.1 . Si el SBR $>1$, entonces el coeficiente $b$ deberá ser ajustado a valores pequeños para alcanzar la calidad de detección máxima posible, sin importar la diferencia entre medias, como se puede ver en la gráfica 3. Ahora veremos los cambios en la calidad de detección cuando cambiamos la potencia de ruido del canal $w$, con el coeficiente $b=0.05$, y corr $=0$.

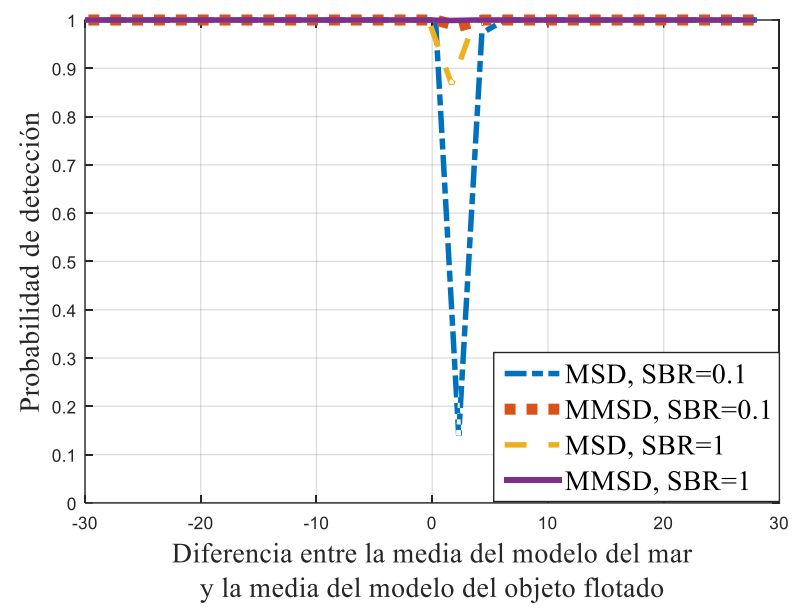

Gráfico 4 Probabilidad de detección vs diferencia entre la media del modelo del mar y la media del objeto flotado. corr $=0.9, w=1, b=0.05$

Fuente: edición del autor. Gráfico extraído de MATLAB

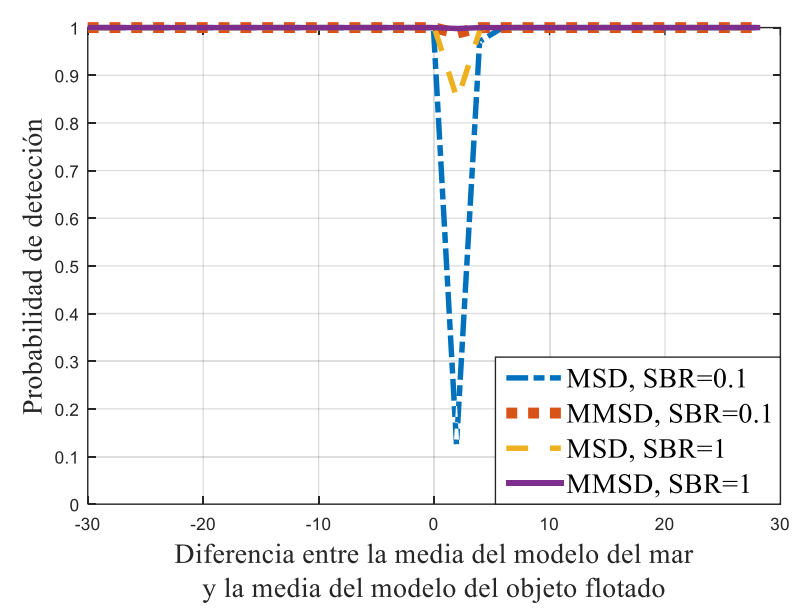

Gráfico 5 Probabilidad de detección vs diferencia entre la media del modelo del mar y la media del objeto flotado. corr $=0.9, w=0.01, b=0.05$

Fuente: edición del autor. Gráfico extraído de MATLAB

De acuerdo con los gráficos 4 y 5 , la calidad de detección de ambos detectores no se ve afectada por los cambios en la potencia del ruido del canal entre los límites establecidos en $w=0.01$ (grafico 5), $w=0.1$ (grafico 3), $\mathrm{y}$ $w=1$ (grafico 4). Por lo tanto, se puede realizar detección en los objetos flotados en la superficie del mar cuando el nivel de ruido del canal es suficientemente grande. Esto significa, que la distancia entre el objeto y el mar, y el medio ambiente, no afecta la calidad de detección.

A continuación, veremos lo que sucede con la calidad de detección al cambiar el factor de correlación corr. 


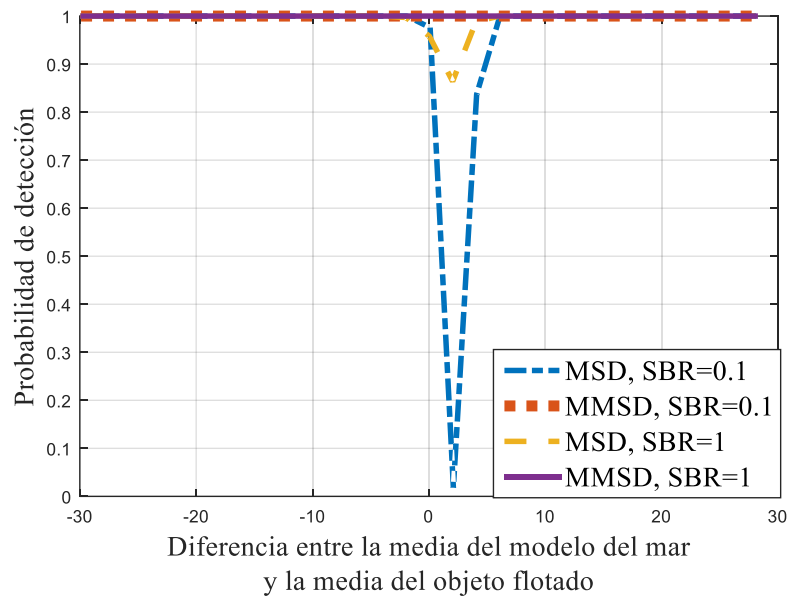

Gráfico 6 Probabilidad de detección vs diferencia entre la media del modelo del mar y la media del objeto flotado. corr $=0.7, w=0.1, b=0.05$

Fuente: edición del autor. Gráfico extraído de MATLAB

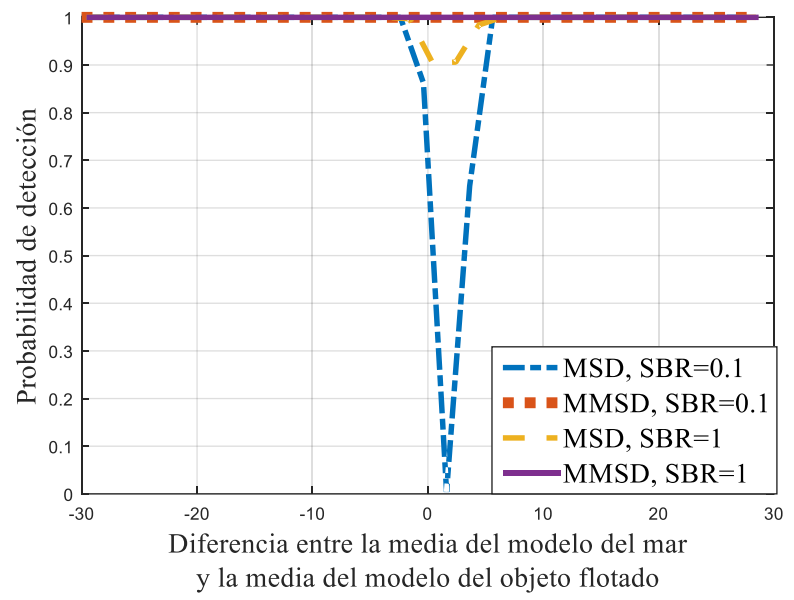

Gráfico 7 Probabilidad de detección vs diferencia entre la media del modelo del mar y la media del objeto flotado. corr $=0.5, w=0.1, b=0.05$

Fuente: edición del autor. Gráfico extraído de MATLAB

De acuerdo con el gráfico 3 (corr $=$ $0.9)$, gráfico $6(\mathrm{corr}=0.7)$ y gráfico $7(\mathrm{corr}=$ 0.5), el detector MMSD siempre alcanza el valor máximo de calidad de detección (la probabilidad de detección cerca de 1). En el caso del detector MSD, este alcanza la calidad de detección deseada cuando el valor de SBR es mayor o igual a 1. Si SBR <1, entonces el detector MSD tendrá un buen desempeño solo si existe diferencia entre las medias de los modelos. Por lo que podemos decir que la se puede realizar la detección de objetos en la superficie del mar, sin importar el nivel de correlación que este tenga.

Ahora analizaremos qué pasa con la calidad de detección cuando la ventana de análisis no coincide completamente con el modelo del objeto flotado, como se muestra en la figura 7 y 8

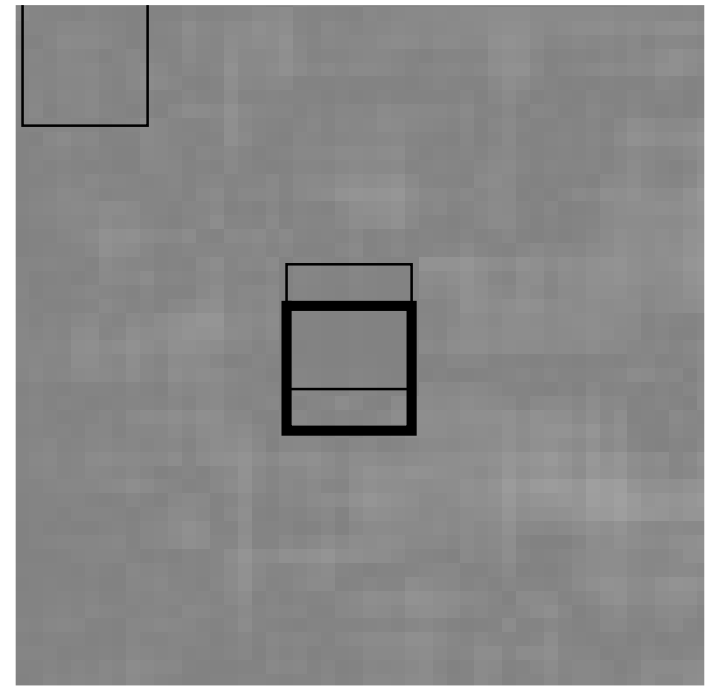

Figura 7 Modelo del objeto flotado de tamaño (primera imagen) $10 \times 10$, dentro del modelo de la superficie del mar de tamaño $50 \times 50$, con ventana de análisis coincidente $70 \%$ con el modelo del blanco. Modelo del mar: $\mu=140, \sigma^{2}=16$, corr $=0.9$. Modelo del objeto flotado: $\mu_{s}=140, \sigma_{s}^{2}=3$

Fuente: Edición del autor. Figura extraída de MATLAB

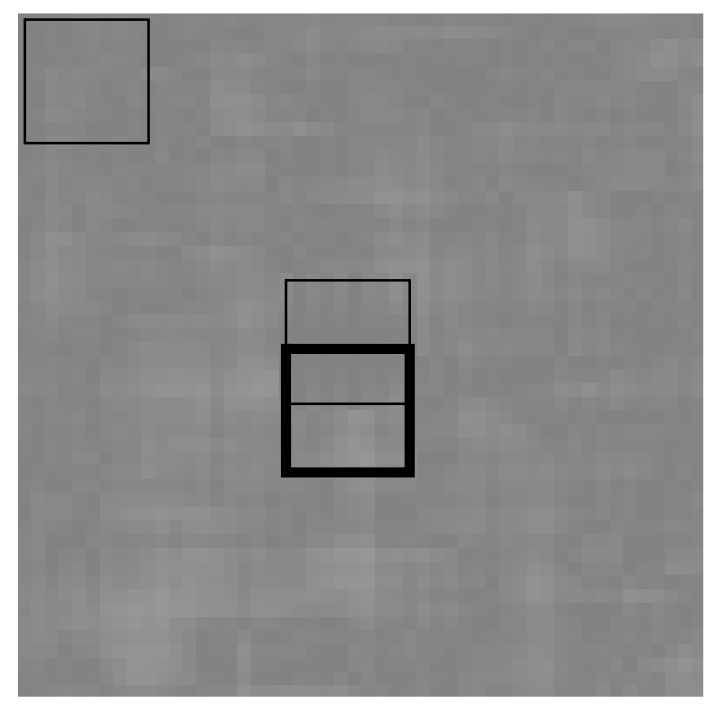

Figura 8 Modelo del objeto flotado de tamaño (primera imagen) $10 \times 10$, dentro del modelo de la superficie del mar de tamaño $50 \times 50$, con ventana de análisis coincidente $50 \%$ con el modelo del blanco. Modelo del mar: $\mu=140, \sigma^{2}=16$, corr $=0.9$. Modelo del objeto flotado: $\mu_{s}=140, \sigma_{s}^{2}=18$

Fuente: Edición del autor. Figura extraída de MATLAB.

Mostraremos el gráfico correspondiente para los modelos mostrados en las figuras 7 y 8 .

PAREDES-PEREZ, Ana Karen, GOLIKOV, Víctor y ALAZKI, Hussain. Detección óptica en 3D de un objeto flotante en una superficie marina agitada. Revista de Invención Técnica. 2020 


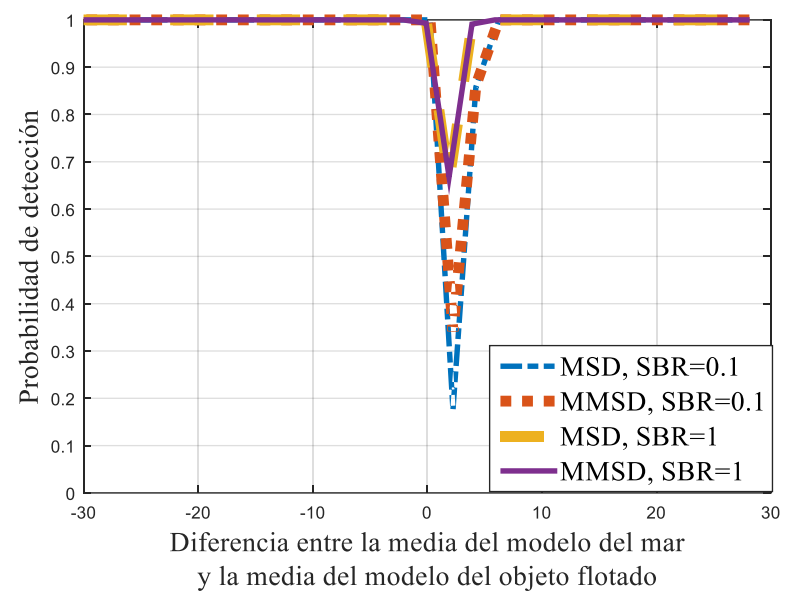

Gráfico 8 Probabilidad de detección vs diferencia entre la media del modelo del mar y la media del objeto flotado. corr $=0.9, w=0.1, b=0.05$. Ventana de análisis coincide $70 \%$ con el modelo del objeto

Fuente: edición del autor. Gráfico extraído de MATLAB

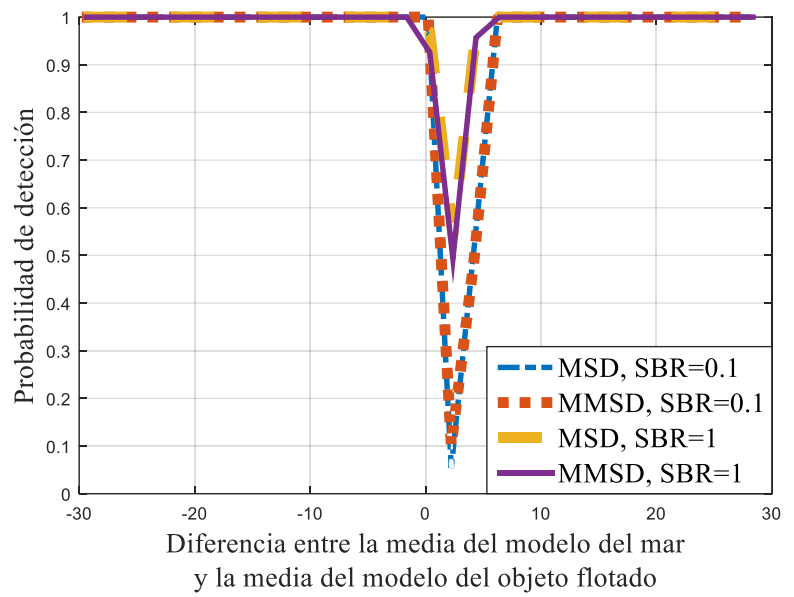

Gráfico 9 Probabilidad de detección vs diferencia entre la media del modelo del mar y la media del objeto flotado. corr $=0.9, w=0.1, b=0.05$. Ventana de análisis coincide $50 \%$ con el modelo del objeto

Fuente: edición del autor. Gráfico extraído de MATLAB

De acuerdo con los gráficos 8 y 9 , se desempeña de manera casi igual cuando la ventana de análisis no coincide completamente con el modelo del objeto flotado. Notamos que los detectores mejoran su calidad de detección, cuando la relación señal-a-fondo SBR crece. Y que, en ambos casos ( $\mathrm{SBR}=0.1$ y $\mathrm{SBR}=1)$, los detectores llegan a la calidad de detección deseada cuando existe una diferencia entre los contrastes del modelo del fondo y el modelo del objeto. Cuando la ventana de análisis no coincide completamente con la posición del objeto de interés, la calidad de detección de ambos detectores baja.

\section{Agradecimientos}

A la Universidad Autónoma del Carmen.

\section{Conclusiones}

Los modelos de reflexiones de luz desde la superficie del mar y desde un objeto flotado se basan en proceso Gaussiano aleatorio para la superficie del mar, y un proceso determinístico desconocido a priori con número de armónicos limitado y pequeño para el modelo del objeto.

La selección de los modelos se basa en trabajos anteriores como en (Blostein \& Huang, 1991) y (Gnanadesikan, 2011) para el modelo de reflexiones de luz desde la superficie del mar, y (Scharf, 1991) en el caso del modelo de las reflexiones de luz desde el objeto flotado como un proceso determinístico desconocido a priori. El modelo de las reflexiones desde los blancos flotados contienen cierto numero de armónicos de frecuencias bajas con amplitudes a priori desconocidos. Hay que seleccionar número de frecuencia más alta $p$ a priori, y para esto nos basamos en experimentos con objetos flotados reales en superficie del mar agitada como en (Golikov, Rodriguez Blanco, \& Lebedeva, 2016) y (Golikov, Lebedeva, \& Rodriguez Blanco, 2016), donde observamos que $p$ puede tomar valores aproximados entre 3 y 7 para las camaras con velocidad 25-30 cuadros/seg.

Los detectores MSD y MMSD requieren información a priori sobre matriz de covarianza $\boldsymbol{R}$ de luz reflejada desde superficie marina y su varianza $\sigma_{0}^{2}$. También hay que seleccionar $p$ y establecer umbrales para cada detector por separado. Inmediatamente después de establecer el umbral hay que iniciar el proceso de detección.

Las condiciones en estadística clásica: MSD requiere la misma información a priori: $\boldsymbol{R}$, $\sigma_{0}^{2}, p$, y calcular el umbral.

La diferencia entre MSD y MMSD es que el MMSD tiene un segundo término logarítmico (como se muestra en la ecuación (8)) sensible a la matriz de covarianza de las señales recibidas. Cuando el MMSD recibe las señales de las reflexiones de luz desde el mar con ruido de canal, el segundo término calcula el valor cerca de 0 , y cuando hay el blanco flotado y ruido del canal (puede contener también parcialmente un parte de energía que refleja el mar) el segundo término aumenta mucho su valor y el detector MMSD alcanza el umbral. 
La calidad de detección de ambos detectores depende de la diferencia entre los parámetros estadísticos de los modelos de las reflexiones desde la superficie del mar y la superficie del objeto de interés. Cuando los parámetros son iguales (la media y la varianza) el detector clásico MSD no alcanza la calidad de detección deseada.

El detector MMSD tiene probabilidad de detección que alcanza valor casi máximo posible cuando los parámetros estadísticos de las reflexiones desde el mar y el objeto flotado son iguales. Esto se puede explicar que el MMSD es sensible con diferencia de ancho de banda del espectro de reflexiones desde la superficie del mar y del objeto.

Las investigaciones presentadas en este artículo muestran que el detector MMSD, tiene una perspectiva buena de implementar en sistemas de detección de los objetos flotados en la superficie del mar.

\section{Referencias}

Blostein, S., \& Huang, T. (1991). Detecting Small, Moving Objects in Image Sequences Using Sequential Hypothesis Testing. IEEE Transactions on Signal Processing, 6.

Chacon Murguia, M. I., Sandoval Rodriguez, R., $\&$ Arias Enriquez, O. (2012). Human gait feature extraction including a kinematic analysis toward robotic power assistance. International Journal of Advanced Robotic Systems, 68.

Gnanadesikan, R. (2011). Methods for Statistical Data Analysis of Multi-variate Observations. John Wiley \& Sons.

Golikov, V., \& Lebedeva, O. (2013). Adaptive detection of subpixel targets with hypothesis dependent background power. IEEE Signal Processing Letters, 751-754.

Golikov, V., Lebedeva, O., \& Rodriguez Blanco, M. (2016). GLRT subspace detection of multipixel targets with known and unknown spatial parameters in presence of signal-dependent background power. Computers \& Electrical Engineering, 38-48.
Golikov, V., Lebedeva, O., Castillejos Moreno, A., \& Ponomaryov, V. (2011). Asymptotically optimum quadratic detection in the case of subpixel targets. IEICE Transactions on Fundamentals of Electronics, Communications and Computer Sciences, 1786-1792.

Golikov, V., Rodriguez Blanco, M., \& Lebedeva, O. (2016). Robust multipixel matched subspace detection with signal-dependent background power. Journal of Applied Remote Sensing, 10.

Healey, G., \& Slater, D. (1999). Models and methods for automated material identification in hyperspectral imagery acquired under unknown illumination and atmospheric conditions. IEEE Transactions on Geoscience and Remote Sensing, 1966-1971.

Kimura, T., Ohashi, M., Crailsheim, K., Schmickl, T., Okada, R., \& Ikeno, H. (2012). Tracking of Multiple Honey Bees on a Flat Surface. Fifth International Conference on Emerging Trends in Engineering and Technology, 36-39.

Manolakis, D., \& Shaw, G. (2002). Detection algorithms for hyperspectral imaging applications. IEEE signal processing magazine, 29-43.

Osorio, R., Lopez Juarez, I., Pena, M., Lomas, V., Lefranc, G., \& Savage, J. (2015). Surveillance System Mobile Object Using Segmentation Algorithms. IEEE Latin America Transactions, 2441-2446.

Scharf, L. L. (1991). Statistical Signal Processing. Addison-Wesley.

Siqueira, D., \& Machado, A. (2016). People Detection and Tracking in Low Frame Rate Dynamic Scenes. IEEE Latin America Transactions, 6 .

Soni, T., Zeidler, J., \& Ku, W. (1993). Performance evaluation of 2-D adaptive prediction filters for detection of small objects in image data. IEEE Transactions on Image processing, 327-340.

Wang, Z., Liao, K., Xiong, J., \& Zhang, Q. (2014). Moving object detection based on temporal information. IEEE Signal Processing Letters, 1403-1407. 